\title{
A influência da relação afetiva e experiencial no processo de criação de lealdade: uma análise do mercado de telefonia móvel no Brasil
}

\section{The influence of affective and experiential relationship in the process of loyalty creation. An analysis in the mobile phone market in Brazil}

\author{
Flavio Vinhal Neves ${ }^{1}$ \\ Flávio Santino Bizarrias ${ }^{2}$ \\ Jussara Goulart da Silva ${ }^{3}$ \\ Marlette Cassia Oliveira Ferreira ${ }^{4}$
}

\section{Resumo}

As inovações em tecnologia móvel trouxeram mais dinâmica ao dia a dia. O uso de celulares particularmente se incorporou ao modo de vida das pessoas, permitindo que experiências de vida e consumo em serviços sejam cada vez mais intensas e dinâmicas. Ao mesmo tempo, as marcas que fornecem estes serviços se incorporaram à identidade dos indivíduos como forma de expressão do self. Este artigo tem por finalidade analisar as relações de experiência com as marcas em serviços, as relações afetivas com as marcas e as respostas do consumidor em termos de lealdade no setor de telefonia móvel brasileiro. Foi realizado um levantamento de campo com 126 estudantes consumidores de serviços de telefonia móvel. Os dados foram analisados por meio de modelagem de equações estruturais. Identificou-se

1 Graduação em Marketing pela Universaidade Nove de Julho - UNINOVE. Afiliação: UNINOVE. Brasil. Lattes: http://lattes.cnpq.br/1428850336639554 Email: flavionevesmkt@gmail.com

2 Doutorando em Administração de Empresas pela Universidade Nove de Julho - UNINOVE. Afiliação: UNINOVE. Brasil. Lattes: http://lattes.cnpq.br/1785231272545956 Email: flavioxsp@ hotmail.com

3 Doutoranda em Administração na Universidade Nove de Julho - UNINOVE. Afiliação: UNINOVE e Universidade Federal de Uberlândia / UFU. Lattes: http://lattes.cnpq.br/2105942822043040 Brasil. Email: jussaragoulart@pontal.ufu.br

4 Doutora em Administração pela Universidade Nove de Julho - UNINOVE. Afiliação: Instituto Federal de Educação, Ciência e Tecnologia de São Paulo - IFSP/Caraguatatuba. Brasil. Lattes: http://lattes.cnpq.br/2937308838723238 Email: marlettecassia@gmail.com 
o amor à marca como construto de maior influência sobre a lealdade em relação à experiência com a marca no âmbito do mercado de telefonia móvel. Este resultado contribui para uma melhor compreensão do relacionamento dos consumidores com marcas e serviços. Por fim, são discutidas implicações teóricas e gerenciais, bem como sugestões de estudos futuros.

Palavras-chave: Marca. Relação afetiva. Relação experiencial. Lealdade. Telefonia móvel.

\section{Abstract}

Innovations in mobile technology have brought more dynamics to the day-to-day. The use of cell particularly if incorporated into the way of life, allowing life experiences and consumer services are increasingly intense and dynamic. At the same time, the brands that provide these services are incorporated to the identity of individuals as a form of self expression. This article aims to analyze the relationship of brand experience in service, and emotional relationships with brands, and consumer responses in terms of loyalty in the Brazilian mobile industry. a field survey with 126 students consumers of mobile services was conducted. Data were analyzed using structural equation modeling. It identified the love of the brand as the most influential construct on loyalty to the brand experience in the mobile market. This result contributes to a better understanding of their relationship with brands and services. Finally, theoretical and managerial implications are discussed as well as suggestions for future studies.

Keywords: Brand. Affective relations. Experiential relations. Loyalty. Mobile phone.

\section{Introdução}

A transação pura e simples com consumidores, a troca de produtos por valores monetários, já não permite sucesso às organizações em longo prazo. A maximização da utilidade em uma relação de consumo não é o objetivo principal do consumidor. A competição extrema e as diversas mudanças no comportamento dos consumidores indicam a importância de estabelecer vínculos mais fortes com eles. Uma alternativa seguida pelas organizações é marketing de relacionamento (GRöNROOS, 1996), que desenvolve mecanismos para um maior encantamento dos clientes dentro de uma economia de experiência (PINE; GILMORE, 1998). Os consumidores não enxergam nos produtos e serviços apenas seu caráter funcional, eles consideram também o significado que as 
marcas possuem em suas vidas (HIRSCHMAN; HOLBROOK, 1982; LEVY, 1959). As coisas que adquirem, e as marcas em particular, passaram, então, a representar um elemento com o qual desenvolvem relações afetivas (AHUVIA; BATRA; BAGOZZI, 2009), ajudando-os a compreender o mundo à sua volta, e a estabelecerem uma identidade ampliada (BELK, 1998).

O relacionamento das pessoas com as organizações é, em grande medida, feito por meio das marcas, as quais representam a organização e suas ofertas, distinguindo essa oferta de uma concorrente (AAKER; JOACHIMSTHALER, 2007). O relacionamento com as marcas (FOURNIER, 1998) se estabeleceu como uma teoria que permite compreender as nuances do papel das marcas na vida das pessoas. Esta relação pessoa-marca-pessoa pode ser carregada de afeto quando o consumidor amplia sua visão de si mesmo e nutre sentimentos em relação a ao objeto (AHUVIA; BATRA; BAGOZZI, 2009; BELK, 1998). O afeto do consumidor por uma marca pode se assemelhar aos sentimentos existentes entre as pessoas nos relacionamentos interpessoais. 0 construto "amor à marca" procura capturar esta ligação emocional intensa do relacionamento do consumidor com as marcas (CARROL; AHUVIA, 2006), propondo que esta seja parte daquele, como a busca da integração de duas identidades em uma só. Marca e consumidor como uma só coisa (WALLACE; BUIL; CHERNATONY, 2014).

A relação pessoa-marca-pessoa envolve, ao mesmo tempo, sensações, sentimentos, aspectos cognitivos e respostas comportamentais aos estímulos da marca, isto é, trata da "experiência com a marca" (BRAKUS; SCHMITT; ZARANTONELLO, 2009). Este conceito observa que as marcas propiciam experiências aos consumidores em três dimensões: a intelectual, a afetivo-sensorial e comportamental nos encontros que o consumidor possui com ela. Os consumidores utilizam produtos e serviços para obter experiências de consumo que irão refletir seu relacionamento com a marca. Este construto oferece a perspectiva de integrar tanto uma dimensão composta de afeto quanto outra caracterizada pela análise e o raciocínio. 
O relacionamento do consumidor com as marcas se inicia com seu primeiro contato com o serviço ou produto e, à medida que este relacionamento se intensifica, esperamos que a afetividade cumpra um papel mediador entre as experiências de serviço, mais cognitivas, e as respostas dos consumidores, como a lealdade.

Esta lealdade pode ser representada pela recompra, mas também por atitudes favoráveis em relação à marca (OLIVER, 1999). Ambos os construtos, amor à marca e experiência com a marca, suscitam lealdade como resposta dos consumidores. Possuir uma relação afetiva e cognitiva intensa com uma marca gera mais lealdade (CARROL; AHUVIA, 2006; NYSVEEN et al., 2013). Um setor da economia que tem se destacado, no Brasil, por sua evolução econômica (SILVA; SANTOS, 2015) e por apresentar características experienciais e de ligação afetivocognitiva com os consumidores é o de serviços de telefonia móvel. As pessoas têm integrado os serviços móveis em suas vidas de tal forma que observamos intensos sentimentos em relação às marcas dos fornecedores desses serviços, bem como uma grande busca por experiências (BRAGAZZI; DEL FUENTE, 2014).

Com base nestas considerações, se estabelece a seguinte questão de pesquisa: Qual a influência do amor à marca e da experiência com a marca sobre a lealdade no setor de telefonia móvel no Brasil? A relação entre estes construtos permite um melhor entendimento das relações afetivas e experienciais dos consumidores com as marcas e suas respostas atitudinais. Não se observam muitos trabalhos neste sentido (ALBERT; MERUNKA, 2015; NYSVEEN et al., 2013), tampouco outro trabalho que tenha realizado a utilização destes construtos em conjunto ou que possa avaliar ainda a afetividade como mediadora de processos cognitivos e respostas do consumidor.

É relevante para pesquisadores, e também para profissionais de marketing, compreender como essas diferentes abordagens teóricas, calcadas em afeto e aspectos cognitivos, influenciam as respostas dos consumidores. Do ponto de vista teórico, este trabalho contribui, portanto, para a discussão acerca da influência dos processos afetivos 
e cognitivos sobre o comportamento do consumidor, sugerindo e comprovando também a mediação do amor à marca para a relação entre processos experienciais e a lealdade, ampliando estudos anteriores para este construto que utilizaram variáveis cognitivas como mediadoras da lealdade (MAFFEZZOLLI; SEMPREBON; PRADO, 2014).

Também se observa a contribuição metodológica deste estudo ao se utilizar dois modelos teóricos concorrentes por meio de modelagem de equações estruturais. Desta forma, se permite analisar simultaneamente o fenômeno do relacionamento dos consumidores com as marcas quando seus antecedentes são mais afetivos ou experienciais. As empresas se beneficiam deste estudo, pois se enfatiza a importância do papel das marcas no relacionamento com os consumidores. Os serviços de telefonia móvel estão cada vez mais presentes na vida das pessoas, e devem ser consideradas nas estratégias das empresas tanto as experiências quanto as ligações fundamentalmente afetivas, particularmente as do setor de telefonia móvel.

\section{Referencial teórico}

\subsection{Relacionamento com a marca}

As marcas não têm uma origem bem definida, entretanto, é certo que existem desde tempos antigos (MOORE; REID, 2008), em que os comerciantes diferenciavam seus produtos através dos formatos ou com assinaturas, como em peças de olaria, tijolos e cerâmica, uma forma utilizada para garantir a procedência da mercadoria. Essa estratégia servia para que os consumidores avaliassem as marcas mais reconhecidas e seguras para eventuais trocas comerciais (AAKER; JOACHIMSTHALER, 2007). Após a Revolução Industrial, o seu uso passou a se massificar, levando as organizações a desenvolverem seus produtos/marcas criando e potencializando o crescimento do seu negócio.

As marcas constituem-se como símbolos emocionais e, à medida que se tornam importantes para a vida das pessoas, deixam de representar 
produtos e serviços apenas, passando a refletirem a identidade das pessoas (LEVY, 1959) em uma perspectiva de relacionamento entre os consumidores e as marcas que escolhem, em uma forma muito semelhante aos relacionamentos interpessoais (AGGARWAL, 2009; FOURNIER, 1998). Em outras palavras, reflete como os consumidores pensam e como se sentem em relação à marca, incorporando diversas teorias, construtos e conceitos que podem ser baseados em basicamente duas dimensões: funcional e emocional (FETSCHERIN; HEINRICH, 2014). Algumas necessidades dos consumidores são mais carregadas de aspectos emocionais. As organizações identificaram esta forma de se diferenciar, bem como a academia, e uma nova corrente de pensamento acadêmico e prática emergiu, em que os consumidores avaliam as marcas com base no relacionamento que possuem com elas (FOURNIER, 1998), ampliando sua identidade (BELK, 1988).

Dentro dessa perspectiva teórica, estabelecem-se diversos conceitos que avaliam as relações afetivas do consumidor com a marca. A ligação (brand attachment) (PARK et al., 2006), afeto e confiança na marca (CHAUDHURI; HOLBROOK, 2001), e sua ligação com a personalidade (AAKER, 1997) ou ainda afeto e paixão (THOMSON; MACINNIS; PARK, 2005), são alguns exemplos. De maneira distinta, todas estas abordagens sugerem que é possível estabelecer relações afetivas entre as marcas e as pessoas, semelhantes às relações interpessoais, como as relações baseadas em amor.

\subsection{Conceituando o amor}

De acordo com Sternberg (1997), o amor é um sentimento que pode ser traduzido em uma experiência emocional não universal, construída por interações sociais, podendo ser diferenciada de acordo com a cultura local. Assim, os significados do amor dependem do contexto histórico observado, temporalidade e especificidades culturais. Segundo Hatfield (1986), podemos caracterizar dois tipos de amor: o apaixonado e o companheiro. O amor apaixonado pode ser caracterizado como emocional e bastante passional. Suas demonstrações são muito intensas, 
não havendo muita intimidade e ainda menos compromisso. Predomina a dimensão da paixão. Já o amor companheiro, é um sentimento de exploração de semelhanças. Nessa fase, o indivíduo necessita ter uma companhia, buscando em seus parceiros a reciprocidade de sentimentos a fim de atender suas necessidades afetivas sociais.

Conforme a Teoria Triangular do Amor, de Sternberg (1997), esse sentimento é composto por três elementos básicos: comprometimento, intimidade e paixão. No primeiro elemento, o comprometimento, há dois aspectos, um de curto prazo e outro de longo prazo. Em curto prazo, refere-se à decisão sobre a existência do amor na relação interpessoal, e, em longo prazo, está relacionado ao comprometimento em manter o amor (STERNBERG,1997). O segundo elemento é a intimidade, que se refere à proximidade e aos laços emocionais no relacionamento, ou seja, é um conhecimento privilegiado do que é a privacidade de uma relação interpessoal, normalmente ocultada (STERNBERG, 1997). O terceiro elemento é a paixão, que é a atração física e consumação sexual, ou seja, é o impulso sexual despertando os fenômenos de desejo e do prazer. Estas teorias do amor interpessoal serviram de base para os estudos iniciais que procuraram avaliar o amor manifestado por objetos, dentre eles as marcas.

\subsection{Amor à marca}

O amor à marca é um construto que recebeu bastante atenção na literatura, variando de uma perspectiva mais ligada aos conceitos do amor interpessoal (AHUVIA, 1993; ALBERT; MERUNKA; VALETTEFLORENCE, 2007; ALBERT; VALETTE-FLORENCE, 2010; ALBERT; MERUNKA, 2015; BAUER; HEINRICH; ALBRECHT, 2009; CARROL; AHUVIA, 2006; RAUSCHNABEL L L., 2015) até uma abordagem mais próxima da interação da marca à identidade do consumidor (BATRA; AHUVIA; BAGOZZI, 2012).

De acordo com Carol e Ahuvia (2006), o amor à marca é definido como o grau de apego sentimental e emocional que um consumidor satisfeito tem com relação a uma marca em particular. Este conceito 
atua como um possível instrumento para mensurar o relacionamento do consumidor com a marca. No contexto brasileiro, o construto recebeu algumas abordagens (BIZARRIAS; LOPES, 2014; FANTINI; GONÇALVES FILHO; SOUKI, 2011; MUNIZ; BAPTISTA, 2011; SANTANA; AKEL SOBRINHO, 2008).

Os relacionamentos entre as marcas e os consumidores podem se consolidar de acordo com a eficiência de estímulos de marketing em se obter uma ligação afetiva mais intensa. Na maior parte das vezes, de forma positiva, aumentando sua intimidade com a marca, conectando a estrutura de conhecimento social do consumidor à marca de seu interesse (AAKER; JOACHIMSTHALER, 2007). No âmbito do objeto deste estudo, o aumento de intensidade do relacionamento do consumidor com a marca se justifica na medida em que serviços de telefonia móvel passam a fazer parte da forma como as pessoas organizam seu mundo, e dessa forma, as marcas desses serviços vêm ocupar um espaço no estilo de vida e na identidade ampliada dos consumidores. O papel mediador da afetividade com as marcas então se estabelece, por um lado, pela experiência gerada com os serviços, e, por outro lado, o crescente apego sentimental a um objeto que passa a ser parte do autoconceito do consumidor, influenciando suas respostas às empresas.

As marcas podem ser construídas para que o consumidor se sinta inserido em um determinado grupo social, ou seja, para que se identifique com as pessoas que também utilizam a mesma marca, podendo resultar em maior sentimento de amor pela marca. A marca serve então ao propósito de ampliação da identidade do consumidor (AHUVIA; BATRA; BAGOZZI, 2009). Quanto maior for este relacionamento e de maneira positiva, maior será a resistência a informações negativas sobre a marca, demonstrando confiança e capacidade de perdoar caso ocorra alguma falha da marca com seus consumidores.

\subsection{Experiência com a marca}

As organizações desenvolvem ações para conquistar a atenção de seu consumidor. O que as leva a questionar quais ações a se tomar 
para o produto ou local de compra serem mais atrativos e agradáveis, de uma forma que estimule as percepções sensoriais, emotivas, cognitivas e comportamentais dos consumidores. Brakus, Schmitt, e Zarantonello (2009) desenvolveram um estudo seminal sobre a definição e mensuração da experiência com a marca.

Os autores definem o conceito como respostas internas afetivas, sentimentais e comportamentais do consumidor a estímulos relacionados à marca. A experiência com a marca não significa necessariamente que haja um julgamento, visto que o conceito está relacionado a sentimentos e sensações que são trazidas à tona pela experiência. A experiência com a marca pode resultar em afeto, embora este aspecto seja também parte da experiência com a marca.

Gobé (2010) apresenta um modelo a partir dos estímulos sensoriais que podem ocorrer através dos cinco sentidos, com estímulos sonoros, visuais, gustativos, táteis, olfativos, entre outros, para a construção de uma relação afetiva com a marca e o consumidor. Nos estímulos sonoros, a música é capaz de gerar sentimentos profundos de uma forma rápida e incontrolável. A experiência com a marca se vale de ações no ambiente, desenvolvendo uma relação intensa do consumidor com a marca.

A experiência com a marca procura capturar dimensões afetivas e cognitivas na experiência de consumo. É composta por uma dimensão sensorial/afetiva, que diz respeito às experiências hedônicas no contato com as marcas. Segundo esta visão as experiências são construídas por meio de sensações. Possui ainda uma dimensão comportamental, que representa as respostas conativas do consumidor na interação com as marcas. Segundo essa dimensão, a experiência que adquirimos se manifesta de maneira motora, como parte de nosso modo de funcionamento na interação com o mundo. Respondemos às experiências com comportamentos. Por fim, possui uma dimensão cognitiva, que compreende que a experiência deriva do conhecimento adquirido nas nossas interações (SCHMITT; BRAKUS; ZARANTONELLO, 2015). As marcas desempenham um papel cada vez mais determinante nas experiências de consumo propriamente ditas para estimular estados 
psicológicos positivos nos consumidores (SCHMITT; BRAKUS; ZARANTONELLO, 2015). Estudos têm observado sua aplicação em diversos momentos de contato com o consumidor, como a internet (HÁ; PERKS, 2005), produto (IGLSESIAS L L., 2011), no setor de serviços (NYSVEEN L L., 2013) e varejo (KHAN; RAHMAN, 2015) ou até mesmo quando se observa sua aplicação na marca corporativa (HAMZAH L L., 2014).

\subsection{Lealdade}

Para manter seus clientes leais as organizações investem em marcas como estratégia de relacionamento. A lealdade é um construto bastante observado na literatura e é associada a um alto grau de avaliações e comportamentos positivos em relação a uma organização ou sua oferta (DICK; BASÚ, 1994). Embora seja bastante associada à repetição de um comportamento de compra, se pode observar a lealdade como uma resposta afetiva, cognitiva ou comportamental favorável (CHAUDHURI; HOLBROOK, 2001; OLIVER, 1999; TAM; WOOD; JI, 2009).

A lealdade pode ser entendida como composta de três dimensões, propostas por Dick e Basú (1994). Uma dimensão diz respeito a (1) componentes cognitivos da lealdade, em que informações sobre o objeto de lealdade são mais acessíveis na memória, em que haja congruência com os valores pessoais do consumidor, gerando confiança e clareza de que a marca é bem estabelecida na mente do consumidor. Outro aspecto diz respeito aos (2) aspectos afetivos da lealdade, relacionados a sentimentos, emoções e estados de humor. Por fim, a lealdade é entendida por (3) questões conativas, que se relacionam a aspectos mais comportamentais.

A lealdade possui antecedentes que irão proporcionar respostas positivas nos consumidores. Espera-se que construtos de natureza afetiva possam gerar maior lealdade no consumidor. 


\subsection{Hipóteses e modelo conceitual}

Estudos tem relacionado aspectos de experiência de consumo vivida por consumidores de serviços móveis (LEE L L., 2015) e de tecnologia em geral (LAM; SHANKAR, 2014), e sua relação com a lealdade, porém, em um âmbito mais utilitário ou, ainda, em contextos de relacionamento com a marca quando prevista por outros construtos atitudinais relacionados a ela (VELOUTSOU, 2015). Coadunando essas abordagens, neste estudo se utiliza os construtos "amor à marca" e "experiência com a marca" como antecedentes da lealdade no setor de serviços móveis.

O amor à marca busca refletir os sentimentos positivos que o consumidor possui em relação aos objetos. Estudos sobre o amor à marca já o relacionaram a lealdade como um Lseqüente importante (CARROL; AHUVIA, 2006; KAMAT; PARULEKAR, 2007). O sentimento amoroso pressupõe um estado psicológico positivo (STERNBERG, 1997), dessa forma, esperamos que houvesse uma relação semelhante em relação aos objetos amados (BELK, 1998), em que a relação seja positiva e significante entre o amor à marca e a lealdade $(\mathrm{H} 1)$.

As experiências de consumo procuram gerar sensações, e estados cognitivos e comportamentais positivos para os consumidores, com vistas a um relacionamento de longo prazo (BRAKUS; SCHMITT; ZARANTONELLO, 2009; SCHMITT; BRAKUS; ZARANTONELLO, 2015), embora isto nem sempre ocorra. Dessa forma, esperamos que houvesse uma relação positiva e significante entre a experiência com a marca e a lealdade $(\mathrm{H} 2)$, e da experiência com a marca com o amor à marca $(\mathrm{H} 3)$.

Como as experiências nem sempre signifiquem uma interação favorável, ao contrário das relações amorosas, que são por definição uma manifestação positiva, espera-se que a relação com a lealdade seja mais forte para o construto amor à marca do que para o construto experiência com a marca. 
O modelo conceitual observado na figura 1 representa o objetivo geral deste estudo, e apresenta as relações entre os construtos, analisado por meio de modelagem de equações estruturais.

Figura 1 - Modelo conceitual

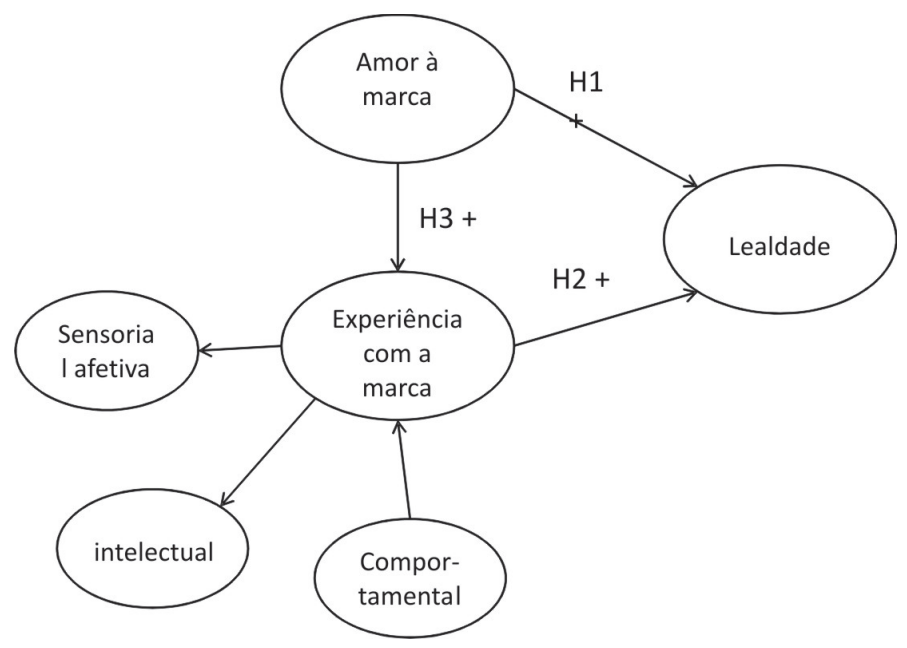

Fonte: Os autores

\section{Método}

\subsection{Caracterização da amostra}

Foram abordados estudantes de administração, em uma pesquisa quantitativa, descritiva, com coorte transversal. A escolha da amostra se deu por conveniência, do tipo não probabilística. $\mathrm{O}$ uso de estudantes se justifica por duas razões. A primeira é o fato de que estudantes de nível superior estão em idade economicamente ativa, sendo responsáveis por suas escolhas de produtos e marcas, na medida em que já buscam sua independência de vida financeira (JOHN, 2009). A segunda é que, ao mesmo tempo, possuem características demográficas e comportamentais heterogêneas representativas do universo característico para este estudo, sendo grandes usuários de serviços de telefonia móvel e demonstrando grande apego às marcas serviços (NICOLACI-DA-COSTA, 2004). 
Para o cálculo do tamanho da amostra se utilizou a regra apresentada por Cohen (1992). Para uma força de $80 \%$, com 5\% de significância, em um modelo com três setas apontando para um construto, e um $R^{2}$ de pelo menos $50 \%$, 38 observações já seriam suficientes com o uso de método de mínimos quadrados parciais.

\subsection{Escalas utilizadas}

As escalas utilizadas foram obtidas com base na revisão da literatura. $\mathrm{O}$ questionário foi apresentado aos respondentes em formato eletrônico. Para a mensuração do amor à marca e lealdade se utilizou a escala de Carrol e Ahuvia (2006) na medida em que estudos anteriores já haviam utilizado estas escalas no contexto brasileiro (FANTINI; GONÇALVES FILHO; SOUKI, 2011; MUNIZ; BAPTISTA, 2011) que passou por um processo de tradução reversa e de comparação com outros instrumentos de mensuração deste construto, se mostrando mais adequada que outros instrumentos de mensuração (BIZARRIAS; LOPES, 2014). Para mensuração da experiência com a marca se utilizou a escala de Brakus, Schmitt, e Zarantonello (2009), adaptada para o cenário brasileiro por Semprebon (2011). Procurou-se guardar o sentido das assertivas das escalas originais.

\subsection{Procedimentos de campo}

A estratégia de coleta de dados escolhida foi um survey realizado entre junho e julho de 2013, com alunos de uma grande universidade na cidade de São Paulo, por meio de questionários aplicados por meio eletrônico. Desta forma, podemos classificar a amostra como não probabilística por conveniência (HAIR, 2010; MALHOTRA, 2009). Quanto à sua natureza este estudo é exploratório.

\subsection{Critérios para análise dos dados}

Para a realização da análise dos resultados, foi efetuada uma Análise Fatorial Confirmatória (AFC) por meio de modelagem de 
equações estruturais (ou SEM, structural equation modeling), para se testar as relações entre as variáveis do estudo. Para a SEM foi utilizado o método de mínimos quadrados parciais (Partial Least Square, PLS) com base em matriz de correlação, dada a possibilidade de não ocorrer a normalidade dos dados, o tamanho reduzido da amostra e o objetivo de previsão dos efeitos (CHIN, 1998; HAIR JR L L., 2014). Se utilizou o software SmartPLS 2.0M3 (RINGLE; WENDE, 2010).

Os primeiros critérios de análise dizem respeito à confiabilidade e validade dos construtos do modelo. Para a confiabilidade do modelo se observou o Alpha de Cronbach $(\geq 0,6)$ e comunalidade $(\geq 0,7)$ dos construtos. Os critérios para aceitação da SEM foram a análise de validade convergente e discriminante. A validade convergente observa a proporção em que uma medida (uma assertiva) se correlaciona positivamente com outras medidas de mensuração do mesmo construto, e que conjunturas procuram explicá-lo. Para isso, se observa as cargas fatoriais dos itens acima de 0,7, e consequentemente a variância média extraída (AVE) do construto acima de 0,5 (mais de 50\% explicado pelos itens que o mensura).

A validade discriminante indica na medida em que os construtos analisados são diferentes. Ou seja, observa se está medindo aquilo que se pretende medir de fato. Esta condição foi testada por meio da análise da raiz quadrada da AVE, por meio dos indicadores de correlação entre os construtos, além da carga fatorial dos itens, devendo ser maiores em suas respectivas variáveis do que nos demais construtos (crossloadings). Também se identifica a validade discriminante quando a raiz quadrada da AVE do construto for superior a qualquer correlação deste com os outros construtos do modelo (CHIN, 1998). Ainda para observação da qualidade do modelo proposto foi considerado o índice de adequação do modelo (Goodnes of Fit, GoF), obtido por meio da média geométrica entre o $R^{2}$ médio (adequação do modelo estrutural) e a AVE média. Este indicador é considerado adequado, nas ciências sociais aplicadas quando acima de 0,36 (HAIR JR, L L. 2014). 
De maneira complementar, como sugerido por Hair Jr L L. (2014) se observou os indicadores de acurácia $\left(Q^{2}\right.$, deve ser superior a 0$)$, e poder previsibilidade do construto para o modelo ( $\mathrm{f}^{2}$, em que indicadores de $0,15,0,36$ ou superior a 0,45 são considerados fraco, médio ou forte, respectivamente). Para o teste das hipóteses se observou a significância das relações entre as variáveis com o uso do bootstrapping para o teste de reamostragem dos dados. Valores de significância do teste $t$ de student $\mathrm{t} \geq 1,96$ (ou $\mathrm{p}$-valor $\leq 0,05$ ) foram considerados aceitos (HAIR JR, L L. 2014).

A mediação afetividade foi verificada por meio do teste de Sobel (1982), ao se comparar o coeficiente de caminho e erro padrão da relação direta entre a variável independente (relacionamento com a marca) e a variável dependente (lealdade), com o coeficiente de caminho e erro padrão da relação da variável mediadora (amor à marca) com a variável dependente. A mediação testa o efeito indireto de uma variável independente sobre outra dependente em razão do efeito de uma terceira variável (mediadora). É considerado significante o efeito quando o teste $\mathrm{t}$ de student apresentar $\mathrm{t} \geq 1,96$ (ou $\mathrm{p}$-valor $\leq 0,05$ )

\section{Análise e discussão dos resultados}

\subsection{Amostra}

Foram abordados 126 estudantes. A amostra válida resultou na totalidade dos respondentes, sem dados faltantes. Deste total, 66 respondentes eram mulheres (52\%) e 60 homens (48\%). Os alunos são da área de Ciências Gerenciais. São predominantemente heavy users de celular, ou seja, 79 dos respondentes (61\%). Outros 37 respondentes (30\%) são middle users, e 10 respondentes (8\%) são low users. Os respondentes ( $n=74$ ou $58,8 \%$ ) declararam a qualidade do sinal, preço do plano ( $n=61$ ou $50,8 \%$ ) e a velocidade da internet $(n=62$ ou $51,7 \%$ ) como os principais aspectos para o relacionamento com a marca. A média de idade foi de 27 anos, com 71,6\% com nível superior completo ou em andamento, $14 \%$ com pós-graduação completa ou em andamento, e $14,4 \%$ calouros. 


\subsection{Modelagem de equações estruturais}

Foi observada a validade convergente e discriminante do modelo, além de indicadores de ajuste até se chegar ao modelo estrutural final. Após três rodadas de testes, com eliminação de itens que não satisfizeram os critérios estabelecidos, se alcançou o modelo estrutural final. O modelo testado apresentou AVEs superiores ao mínimo exigido e indicadores de alpha de Cronbach, confiabilidade composta e comunalidade adequados, além de GoF significante. Estes resultados podem ser vistos nas Tabelas 1 e 2 . A validade discriminante também foi observada por meio dos crossloadings (Apêndice A) com a carga fatorial dos itens mais elevada nas suas respectivas variáveis.

Tabela 1 - Indicadores de adequação do modelo - $\mathrm{GoF}=0,803$

\begin{tabular}{l|c|c|c|c|c|c}
\hline Construto & AVE & $\begin{array}{c}\text { Confiabilidade } \\
\text { composta }\end{array}$ & $\mathbf{R}^{2}$ & $\begin{array}{c}\text { Alpha de } \\
\text { Cronbach }\end{array}$ & Comunalidade & Redundância \\
\hline Amor a marca & 0,815 & 0,964 & 0,752 & 0,955 & 0,815 & 0,611 \\
\hline Comportamental & 0,829 & 0,907 & 0,788 & 0,794 & 0,829 & 0,653 \\
\hline Intelectual & 0,853 & 0,921 & 0,852 & 0,828 & 0,853 & 0,726 \\
\hline Lealdade & 0,782 & 0,935 & 0,762 & 0,907 & 0,782 & 0,539 \\
\hline Sensorial afetiva & 0,715 & 0,883 & 0,903 & 0,8 & 0,715 & 0,643 \\
\hline
\end{tabular}

Fonte: Resultados da Pesquisa.

Tabela 2 - Validade discriminante

\begin{tabular}{l|c|c|c|c|c}
\hline Construto & $\begin{array}{c}\text { Amor a } \\
\text { marca }\end{array}$ & Comportamental & Intelectual & Lealdade & $\begin{array}{c}\text { Sensorial } \\
\text { afetiva }\end{array}$ \\
\hline Amor a marca & $0,903^{*}$ & & & & \\
\hline Comportamental & 0,683 & $0,910^{*}$ & & & \\
\hline Intelectual & 0,84 & 0,729 & $0,924^{*}$ & & \\
\hline Lealdade & 0,863 & 0,67 & 0,751 & $0,884^{*}$ & \\
\hline Sensorial afetiva & 0,85 & 0,767 & 0,823 & 0,807 & $0,846^{*}$ \\
\hline
\end{tabular}

${ }^{*}$ Raiz quadrada da AVE

Fonte: Resultados da Pesquisa. 
A análise estrutural indicou caminhos significativos entre os construtos do modelo como demonstrado na Tabela 3, confirmando as hipóteses estabelecidas.

Tabela 3 - Coeficientes estruturais do modelo

\begin{tabular}{c|c|c|c|c|c|c|c|c}
\hline Hipótese & Sentido & Relações & $\begin{array}{c}\text { Coeficiente } \\
\text { original }\end{array}$ & $\begin{array}{c}\text { Média das 200 } \\
\text { subamostras }\end{array}$ & $\begin{array}{c}\text { Erro } \\
\text { padrão }\end{array}$ & Teste t & p-valor & Resultado \\
\hline $\mathrm{H} 1$ & Positivo & $\begin{array}{c}\text { Amor a marca } \\
\text { Lealdade }\end{array}$ & 0,631 & 0,635 & 0,09 & 6,984 & 0,001 & Confirmada \\
\hline $\mathrm{H} 2$ & Positivo & $\begin{array}{c}\text { Exp.com a marca } \\
\rightarrow \text { Lealdade }\end{array}$ & 0,267 & 0,267 & 0,092 & 2,888 & 0,005 & Confirmada \\
\hline $\mathrm{H} 3$ & Positivo & $\begin{array}{c}\text { Exp.com a marca } \\
\rightarrow \text { Amor a marca }\end{array}$ & 0,867 & 0,868 & 0,021 & 42,388 & 0,001 & Confirmada \\
\hline- & $-\begin{array}{c}\text { Exp.com a marca } \\
\rightarrow \text { Sensorial } \\
\text { afetiva }\end{array}$ & 0,95 & 0,95 & 0,01 & 98,968 & 0,001 & - \\
\hline- & $\begin{array}{c}\text { Exp.com a marca } \\
\rightarrow \text { Intelectual }\end{array}$ & 0,923 & 0,924 & 0,015 & 63,48 & 0,001 & - \\
\hline
\end{tabular}

Fonte: Resultados da Pesquisa.

O modelo estrutural ajustado apresentou relações positivas e significantes $(p<0,1 \%$ e $t>1,96)$ entre todos os construtos, identificados pelo método de reamostragem bootstrapping. O modelo final apresentou uma excelente qualidade de ajuste. O cálculo do GoF, por meio da média geométrica entre os valores médios do $\mathrm{R}^{2}$ e a AVE média ficou em 0.803 , acima da recomendação de um mínimo de 0.36 , nas ciências sociais (HAIR JR L L., 2014).

O modelo estrutural demonstrou que existem relações positivas e significantes entre o amor à marca e a experiência com a lealdade $(\mathrm{H} 1$ : $\left.\Gamma=0.631 ; t_{(126)}=6,984 ; p<0.001\right)$, a experiência com a marca e a lealdade $\left(H 2: \Gamma=0.267 ; t_{(126)}=2,888 ; p=0.005\right)$, e a experiência com a marca e o amor à marca $\left(H 3: \Gamma=0.867 ; t_{(126)}=42,388 ; p<0.001\right)$. A tabela 4 apresenta os resultados de acurácia $\left(\mathrm{Q}^{2}\right)$ e importância do construto para o modelo $\left(f^{2}\right)$. 
Tabela 4 - Acurácia e previsibilidade do modelo

\begin{tabular}{c|c|c}
\hline Construto & $\mathrm{Q}^{2}$ & $\mathrm{f}^{2}$ \\
\hline Amor à marca & 0,53 & 0,707 \\
\hline Experiência com a marca & - & - \\
\hline Comportamental & 0,644 & - \\
\hline Intelectual & 0,731 & - \\
\hline Sensorial afetiva & 0,638 & - \\
\hline Lealdade & 0,56 & - \\
\hline
\end{tabular}

Fonte: Resultados da Pesquisa.

Estes indicadores apontam para a robustez do modelo teórico proposto e para seu poder preditivo da lealdade. Também se pode observar a relação significativa entre amor à marca e a experiência com a marca.

O objetivo deste trabalho foi analisar a influência do amor à marca e experiência com a marca sobre a lealdade, no contexto brasileiro do mercado de celulares. Se considerou este objetivo alcançado, pois todas as hipóteses foram comprovadas, e os resultados permitem uma análise das relações entre as variáveis em um grupo de consumidores capaz de representar grande parte dos consumidores de serviços móveis. Também se considerou alcançado o objetivo deste estudo ao relacionar pela primeira vez o amor à marca e a experiência com a marca como antecedentes da lealdade simultaneamente. Este estudo responde também à carência de estudos sobre a estes construtos no âmbito de serviços (BATRA; AHUVIA; BAGOZZI, 2012; KHAN; RAHMAN, 2015).

Os resultados do modelo estrutural apresentaram caminhos positivos e significativos entre as variáveis propostas explicando $76.2 \%$ da lealdade declarada. Observou-se que a o amor à marca obteve maior influência sobre a lealdade $\left(\Gamma=0.631 ; \mathrm{t}_{(126)}=6,984 ; p<0.001\right)$. Isto sugere que nas relações entre consumidores e marcas, no âmbito dos celulares, a experiência de consumo e sua face mais cognitiva, embora relevante $\left(\Gamma=0.267 ; t_{(126)}=2,888 ; p=0.005\right)$, seja secundária em relação a uma ligação afetiva mais intensa com marca, como esperávamos, pois o amor à marca é um construto de natureza positiva por si só, o que 
não ocorre com as experiências de consumo sempre. Testes adicionais revelaram esse papel mediador relevante do amor à marca para a relação das experiências de consumo com a lealdade (Teste de Sobel=6,912, $p<0,001)$, refletido na importância do construto para o modelo $\left(f^{2}=0,707\right)$, maior que as demais variáveis.

Este estudo contribui para o debate sobre a relação entre afeto e cognição no âmbito do comportamento do consumidor identificando que as relações afetivas com as marcas são mais substanciais no setor de serviços móveis. O amor à marca pode ser observado nas manifestações dos consumidores quando estes sentem grande apego aos objetos e às marcas. Não raro pessoas descrevem o quão dependente psicologicamente são destes equipamentos (NICOLAClDA-COSTA, 2004). A ciência identificou novas patologias relacionadas ao uso intenso dos celulares e dispositivos móveis (BRAGAZZI; DEL PUENTE, 2014). Esta intensa utilização dos recursos móveis em muito se assemelha a um relacionamento interpessoal intenso, carregado de afeto.

A despeito do debate corrente sobre as propriedades psicométricas de mensuração do amor à marca e sua definição (AHUVIA; BAGOZZI, BATRA, 2014; ROSSISTER, 2012), este estudo identificou este construto como relevante na previsão de respostas atitudinais de lealdade. A definição de amor empregada nas relações entre pessoas é utilizada para se aproximar a definição de amor por objetos de atitude como as marcas. Este estudo utilizou esta corrente de pensamento acadêmico acerca do construto Amor à marca, embora reconheçamos que se pode observar o construto "[...] como um sentimento ou um relacionamento" (AHUVIA; BAGOZZI; BATRA, 2014, p. 236). Ao testar empiricamente ambas as visões, este estudo enseja que o debate acadêmico, e seus reflexos na prática das organizações ainda permanece aberto. Para as organizações ambas as abordagens são úteis, na medida em que as marcas dos celulares se incorporam à identidade das pessoas, e seu modo de vida.

Todas as dimensões da experiência com a marca obtiveram uma variância explicada considerável, com destaque pra as dimensões 
sensorial afetiva $\left(R^{2}=90,3 \%, Q^{2}=0,638\right)$, e intelectual $\left(R^{2}=85,2 \%\right.$, $\left.Q^{2}=0,731\right)$, levemente mais elevadas que a dimensão comportamental $\left(R^{2}=78,8 \%, Q^{2}=0,644\right)$, mas da mesma forma, sugerindo uma maior importância das relações afetivas com a marca. No dia a dia as pessoas usam os serviços móveis para organizar suas tarefas e obter entretenimento. Isto leva a um relacionamento diário com as marcas de celulares. Poucos produtos ou serviços estão tão presentes na vida dos consumidores. A experiência é vivida intensamente e estabelece uma conexão afetiva grande. Ao mesmo tempo exige das pessoas o uso de mecanismos cognitivos a todo o momento.

Os serviços móveis são também uma experiência on-line que promove o relacionamento com a marca mesmo que não haja interação entre pessoas. A experiência é estimulada pela marca do celular, como símbolo do conjunto de significados que a experiência diária de uso dos serviços móveis proporciona, com um cunho afetivo intenso. Entretanto, isto não é consenso. Jung e Soo (2012) identificaram que as experiências com a marca não precisam ser sempre afetivas, pois isto depende do objetivo das organizações com um determinado público-alvo. Entendemos que embora esta proposição seja válida, os consumidores irão valorizar mais sua experiência com uma determinada marca sempre que houver afeto envolvido. Ramaseshan e Stein (2014) identificaram que a lealdade é em grande parte prevista por experiências significativas com a marca, particularmente afetivas, corroborando nossos achados. Frank L L. (2014, p. 578) identificaram no setor de telefonia móvel um maior efeito de mecanismos afetivos do que cognitivos na construção da experiência com a marca que gere maior lealdade. Sugerimos que mais estudos sejam realizados em outros contextos para se contribuir ainda mais para o avanço teórico acerca destes construtos.

\section{Conclusão}

Para as organizações, este estudo contribui em ensejar a discussão sobre a importância em se construir relações afetivas entre as marcas e os consumidores na busca pela lealdade. Embora o 
marketing de experiência tenha crescido bastante enquanto prática das organizações parece haver uma prevalência de um relacionamento mais afetivo entre os consumidores e as marcas, particularmente no mercado de telefonia móvel. Isto é coerente com a distinção entre os construtos. A experiência com a marca não significa uma relação sempre positiva entre o consumidor e a marca, mas o amor à marca pressupõe uma relação sempre positiva com a marca. O que apontamos não está em se escolher um dos caminhos, experiência ou afeto para a construção de relacionamentos fortes com os consumidores. Entendemos que ambas as abordagens são compatíveis com o observado, neste estudo, na relação entre os construtos $\left(\Gamma=0.867 ; t_{(126)}=42,388 ; p<0.001\right)$. Este estudo indica que o amor à marca se soma ao conjunto de variáveis antecedentes da lealdade, porém enfatizando as relações afetivas positivas com as marcas, de uma maneira semelhante aos relacionamentos interpessoais sem, no entanto, sem deixar de se abordar a construção de experiências de consumo.

As organizações devem desenvolver experiências de consumo para se diferenciar de seus concorrentes, mas também capturar e satisfazer consumidores mais exigentes. Para os gestores de marketing esse é um desafio considerável, pois pressupõe uma perspectiva de longo prazo, impondo às organizações muitas vezes perdas iniciais. Ponderamos que as experiências de consumo não são de natureza sempre favorável nas relações com os consumidores, como foi observado neste estudo, e esta constatação reforça ainda mais a necessidade de uma gestão bastante próxima da relação do consumidor com a marca. Não basta construir uma marca forte, é preciso acompanhar como o consumidor atribui valor a ela. Propomos então que o Amor à marca seja um caminho. Seguir esta orientação exige inovação das organizações na medida em que se deverá gerir a relação entre a marca e o consumidor numa perspectiva de relacionamentos interpessoais, em que os ganhos nem sempre são equitativos para ambas as partes (LEE, 1988).

Propomos como estudos futuros o uso de técnicas de estudo complementares à abordagem quantitativa dentro de um paradigma interpretativista. Desta forma, se pode aprofundar o entendimento das 
razões que possam levar o consumidor a satisfazer suas necessidades afetivas por meio das marcas, além de permitir que se observe como se relacionam estas necessidades afetivas e de extensão da identidade com as ações mais experienciais desenvolvidas pelas organizações. Em que ponto convergem, e quais as motivações mais profundas para este consumidor.

Por fim, este estudo não se estabelece como uma pesquisa conclusiva sobre os construtos observados. Foi observado o papel mediador das relações afetivas entre consumidores e marcas na construção da lealdade mesmo que haja uma experiência de consumo favorável. Também permitiu que se pudesse avaliar a relação entre eles, em um setor importante na economia e tão presente na vida social das pessoas.

\section{Referências}

AAKER, D. A.; JOACHIMSTHALER, E. Como construir marcas líderes. Porto Alegre: Bookman, 2007. 324p

AAKER, J.. Dimensions of Brand Personality. Journal of Marketing Research.[S.I], v. 34, n.3, p. 347-356, Aug.1997

AGGARWAL, M.. Using relationship norms to understand consumerbrand interactions. In: MACINNIS, D. J.; PARK, C. W.; PRIESTER, J. $\mathrm{R}$ (eds). The Handbook of Brand Relationships. London: New York: M.E. Sharpe, 2009. p 24-42.

\section{AHUVIA, A. C. I Love It! Towards a Unifying Theory of love across} diverse love objects. 1993. 62f. Unpublished Ph.D. Doctoral dissertation. School of Business Administration. Northwestern University, Kellogg School of Management. University of Michigan. Ann Arbor1993

AHUVIA, A.; BATRA, R.; BAGOZZI, R. P. Love, Desire, and Identity. A conditional integration theory of the love of things. In: MACINNIS, D. J.; PARK, C. W.; PRIESTER, J. R (eds). Handbook of Brand 
Relationship. London: New York: Society of Consumer Psychology: M.E. Sharpe, 2009. p. 342-357.

AHUVIA, A.; BATRA, R.; BAGOZZI, R. P. Psychometric vs. C-OAR-SE measures of brand love: A reply to Rossiter. Marketing Letters. [S.I], v.25, n.2, p.235-243, jun. 2014

ALBERT, N.; VALETTE-FLORENCE, P.. Measuring the love feeling for a brand using interpersonal love items. Journal of Marketing Development and Competitiveness. [S.I], v. 5, n. 1, p. 57, 2010

ALBERT, N.; MERUNKA,D.Role of Brand Love in Consumer Brand Relationships. In: FETSCHERIN, M.;HEILMANN,T. (eds).Consumer Brand Relationships:Meaning, Measuring, Managing. London:Palgrave MCMillan, 2015.p. 15-30,

ALBERT, N.; MERUNKA, D.; VALETTE-FLORENCE, P.. The feeling of love toward a brand: concept and measurement. Advances in Consumer Rsearch. NorthAmerican Conference Proceedings, v. 36, p. 300-307, 2009.

BATRA, R.; AHUVIA, A. C.; BAGOZZI, R. P. "Brand Love". Journal of Marketing; [S.I], v.76, n.2, p. 1-16, Mar.2012

BAUER, H., HEINRICH, D., ALBRECHT, C. M.. All you need is love: assessing consumers' brand love. In: KAMIN , M.; MARTIN, I. M. (eds) Proceedings of the American Marketing Association. Summer Educators Conference. Chicago: American Marketing Association, 2009. p. 252-53.

BELK, R. W. Possessions and the Extended Self. Journal of Consumer Research, [S.I], v. 2, n. 15, p. 139-168, 1998.

BIZARRIAS, F. S.; LOPES, E. L. Escalas concorrentes para mensuração do amor à marca: um estudo no contexto nacional. In: Encontro de Marketing da Associação Nacional de Pós-Graduação e Pesquisa em Administração - EMA ANPAD, 6., 2014. Gramado/RS. Anais... Gramado/RS: EMA ANPAD, 2014. p. 1-16. 
BRAGAZZI, N. L.; DEL PUENTE, G. A proposal for including nomophobia in the new DSM-V. Psychology Research and Behavior Management.[S.I], v. 16, n.7, p. 155-160, May 2014.

BRAKUS, J. J.; SCHMITT, B. H.; ZARANTONELLO, L. Brand experience what is it how is measured. Journal of Marketing. [S.I], v. 73, n. 3, p. 52-68, May 2009.

CARROLL, B.; AHUVIA, A. Some antecedents and outcomes of brand love. Marketing Letters. [S.I], v. 17, n. 2, p. 79-89, Apr. 2006

CHAUDHURI, A., HOLBROOK, M. B. The chain of effects from brand trust and brand affect to brand performance: The role of brand loyalty. Journal of Marketing. [S.I], v. 65, n. 2, p. 91-93, Apr. 2001

CHIN, W. W. The partial least squares approach to structural equation modeling. In: MARCOULIDES, G. A. (ed.) Modern methods for business research. Mahwah, NJ, US: LawrenceErlbaum Associates, Inc., 1998. p.295-336.

COHEN, J. A power prime. Psychological Bulletin. [S.I], v. 112, n. 1 , p. 155-159, Jul. 1992

FANTINI, M. J.; GONÇALVES FILHO, C.; SOUKI, G. Q. Antecedentes do amor à marca e seus impactos nas intenções comportamentais de consumidores: um estudo empírico no setor automotivo. Revista Gestão e Planejamento, Salvador, v. 12, n. 1, p. 74-94, jan./jun. 2011.

FETSCHERIN, M.; HEINRICH, D.. Consumer brand relationships: a research landscape. Journal of Brand Management. [S.I], v. 21, n. 5, p. 366-371, 2014.

FOURNIER, S. Consumers and their brands: developing relationship theory in consumer research. Journal of Consumer Research. [S.I], v. 24, n. 4, p. 343-373, 1998.

FRANK, B.; TORRICO, B. H.; ENKAWA, T., SCHVANEVELDT, S. J. Affect versus cognition in the chain from perceived quality to customer 
loyalty: The roles of product beliefs and experience. Journal of Retailing.[S.I], v. 90, n. 4, p. 567-586, Dec, 2014.

GOBÉ, M.. Emotional branding: the new paradigm for connecting brands to people. New York, NY : Allworth Press: Updated and Revised Edition edition, 2010.

GRÖNROOS, C.. Relationship marketing: strategic and tactical implications. Management Decision. [S.I], v. 34, n. 3, p. 5- 14, 1996

HA, H.Y.; PERKS, H.. Effects of consumer perceptions of brand experience on the web: brand familiarity, satisfaction and brand trust. Journal of Consumer Behaviour. [S.I], v. 4, n. 6, p. 438-452, 2005

HAIR J. F, et al.. A primer on partial least squares structural equation modelling (PLS-SEM). 2nd Edition. Thousand Oaks: SAGE Publications, 2014.

Hair, Joseph F., et al. Multivariate data analysis: a global perspective. 7th ed. Upper Saddle River: Prentice Hall, 2009

HAMZAH, Z. L.; ALWI, S. F. S.; OTHMAN, M. N.. Designing corporate brand experience in an online context: A qualitative insight. Journal of Business Research. [S.I], v. 67, n. 11, p. 2299-2310, 2014

HATFIELD, E.; SPRECHER, S. Measuring passionate love in intimate relationships. Journal of Adolescence. [S.I], v. 9, n. 4, p.383-410, 1986.

HIRSCHMAN, E. C.; HOLBROOK, M. Hedonic consumption: emerging concepts, methods and propositions. Journal of Marketing. [S.I], v. 46, n. 3, p. 92-101, 1982.

IGLESIAS, O.; SINGH, J. J; BATISTA-FOGUET, J. M. The role of brand experience and affective commitment in determining brand loyalty. Journal of Brand Management. [S.I], v. 18, n. 8, p. 570-582, 2011 JOHN, D. R.. Stages of consumer socialization: the development of consumer knowledge, skills, and values, from childhood to adolescence. In: HAUGTVEDT, C. P.; HERR, P. M.; KARDES, F. R. 
Handbook of consumer psychology. New York, NY: Lawrence Erlbaum Associates, 2009, p. 297-348.

JUNG, L. H.; SOO, K. M. The effect of brand experience on brand relationship quality. Academy of Marketing Studies Journal. [S.I], v.16, n. 1, p.87-98, 2012.

KAMAT, V. V.; PARULEKAR, A. A. BrandLove: the precursor to loyalty. In: PRIESTER, J. R.; MACINNIS, D. J.; PARK, C. W. (eds). New frontiers in branding: attitudes, attachments, and relationships.

Advertising and Consumer Psychology Conference, 26., 2007, Santa Mônica (EUA). Anais... Santa Mônica: Society for Consumer Psychology, 2007, p. 94-98.

KHAN, I.; RAHMAN, Z. A review and future directions of brand experience research. International Strategic Management Review,[S.I], v. 3, n.1-2, p. 1-14, Jun.-Dec. 2015.

LAM, S. Y.; SHANKAR, V.. Asymmetries in the effects of drivers of brand loyalty between early and late adopters and across technology generations. Journal of Interactive Marketing, [S.I], vol. 28, n. 1, p. 26-42, 2014.

LEE, J. A. Love styles. In: BARNES. M.H.; STERNBERG, R. J. The Psychology of love. New Haven, Conn: Yale University Press.1988. p. 38-67.

LEE, D.; MOON, J.; KIM, Y. J.; YI, M. Y. Antecedents and consequences of mobile phone usability: Linking simplicity and interactivity to satisfaction, trust, and brand loyalty. Information \& Management. vol. 52, n. 3, p. 295-304, Apr. 2015

LEVY, S. J. Symbols for sale. Harvard Business Review.[S.I], v. 37, n.4, p. 117-124, 1959.

MALHOTRA, N.. Marketing research: an applied orientation. 6th ed. NY: Pearson Education, 2009. 
MAFFEZZOLLI, E. C. F; SEMPREBON, E.; PRADO, P. H.

M.Construing loyalty through brand experience: the mediating role of brand relationship quality. Journal of Brand Management. [S.I], v. 21, n. 5, p. 446-458, June, 2014

MOORE, K.; REID, S.. The birth of brand: 4000 years of branding history. Business History. [S.I], v. 50, n. 4, p. 419-432, July, 2008.

MUNIZ, K. M., BAPTISTA, P. P.. O consumidor está amando: a natureza e o potencial do construto do brand love para o estudo do relacionamento entre consumidores e marcas. In: ENCONTRO NACIONAL DA ANPAD,35., 2011, Rio de Janeiro. Anais... Rio de Janeiro: EnANPAD , p. 1-17, 2011.

NICOLACI-DA-COSTA, A. M. Impactos psicológicos do uso de celulares: uma pesquisa exploratória com jovens brasileiros. Psicologia: Teoria e Pesquisa. [S.I], v. 20, n. 2, p. 165-174, Maio-Ago. 2004.

NYSVEEN, H.; PEDERSEN, P.E.; SKARD, S. Brand experiences in service organizations: exploring the individual effects of brand experience dimensions. Journal of Brand Management. [S.I], v.20, n.5, p. 404-423, Apr.2013

OLIVER, R. L. Whence consumer loyalty? Journal of Marketing. [S.I], v. 63, Special Issue, p. 33-44, 1999

PARK, C. W.; MACINNIS, D. J.; PRIESTER, J.. Brand attachment: constructs, consequences, and causes. Boston/Delft: Foundations \& Trends in Marketing.2006. v. 1, n. 3, p. 191-230.

RAMASESHAN, B; STEIN, A. Connecting the dots between brand experience and brand loyalty: the mediating role of brand personality and brand relationships. The Journal of Brand Management, [S.I], v. 21, n. 7-8, p. 664-683, 2014.

RAUSCHNABEL, P. et. al.The Personality of Brand Lovers. In: FETSCHERIN, M.; TOBIAS, H (eds). Consumer Brand 
Relationships:Meaning, Measuring, Managing. London: Palgrave MCMillan, 2015. p.108-122.

RINGLE, C. M.; WENDE, S.; WILL, S. SmartPLS. 2.0 (M3) Beta, Hamburg. 2005. Disponível em: <www.smartpls.de>. Acesso em: ago. 2008

ROSSITER, J. R. A new C-OAR-SE-based content-valid and predictively valid measure that distinguishes brand love from brand liking. Marketing Letters. [S.I], v.. 23, n. 3, p. 905-916, 2012.

SANTANA, É. E. P.; AKEL SOBRINHO, Z.. Um modelo conceitual sobre a influência do amor à marca no comportamento do consumidor no concernente à indústria do futebol. In: ENCONTRO DA ASSOCIAÇÃO NACIONAL DE PÓS-GRADUAÇÃO E PESQUISA EM ADMINISTRAÇÃO - EnANPAD, 32., 2008, Rio de Janeiro. Anais... Rio de Janeiro: ANPAD, p. 1-16, 2011.

SCHMITT, B. H.; BRAKUS, J.; ZARANTONELLO, L .The current state and future of brand experience. Journal of Brand Management. [S.I], v. 21, n. 9, p. 727-733, Dec. 2015.

SEMPREBON, E. Experiência com marca: validação da escala no contexto universitário. In:: Encontro da ANPAD (EnANPAD), 35., 2011, Rio de Janeiro. Anais... Rio de Janeiro: ANPAD, p. 1-17, 2011.

SILVA, C. T. R.; SANTOS, D. F. L. Desempenho financeiro e valor de mercado do setor de telefonia no Brasil. Revista Ciências Administrativas. Fortaleza, v. 21, n. 1, p. 42-67, jan./jun. 2015 SOBEL, M. E. Asymptotic confidence intervals for indirect effects in structural equation models. Sociological Methodology. [S.I], v. 13, p. 290-312, 1982.

STERNBERG, R. J. A triangular theory of love. European Journal of Social Psychology. [S.I], v. 27, n. 3, p. 313-335, 1997.

TAM, L.; WOOD, W.; JI, M. F. Brand Loyalty is not Habitual. In MACLNNIS, D. J.; PARK, C. W.; PRIESTER, J. R. (Eds.). Handbook 
of Brand Relationship. London, UK: Society of Consumer Psychology, p. 43-62, 2009.

THOMSON, M., MACINNIS, D.J., PARK, C.W. The ties that bind: measuring the strength of consumers' emotional attachment to brands. Journal of Consumer Psychology. [S.I], v. 15, n. 1, p. 77-91, 2005 VELOUTSOU, C.. Brand evaluation, satisfaction and trust as predictors of brand loyalty: the mediator-moderator effect of brand relationships. Journal of Consumer Marketing. [S.I], v. 32, n. 6, p.405 - 421, Sep. 2015.

WALLACE, E.; BUIL, I.; CHERNATONY, L. Consumer engagement with self-expressive brands: brand love and WOM outcomes. Journal of Product \& Brand Management. [S.I], v.23, n.1, p. 33-42, Mar. 2014. 


\section{Apêndice A}

Tabela 7 - Crossloadings

\begin{tabular}{c|c|c|c|c|c}
\hline Itens & Amor a marca & Comportamental & Intelectual & Sensorial afetiva & Lealdade \\
\hline am10 & $\mathbf{0 , 8 9 5}$ & 0,552 & 0,702 & 0,729 & 0,786 \\
\hline am2 & $\mathbf{0 , 8 9 4}$ & 0,633 & 0,754 & 0,778 & 0,774 \\
\hline am3 & 0,873 & 0,679 & 0,773 & 0,791 & 0,769 \\
\hline am5 & $\mathbf{0 , 9 0 2}$ & 0,638 & 0,786 & 0,754 & 0,795 \\
\hline am6 & $\mathbf{0 , 9 2 7}$ & 0,601 & 0,756 & 0,773 & 0,776 \\
\hline am9 & $\mathbf{0 , 9 2 6}$ & 0,593 & 0,777 & 0,775 & 0,772 \\
\hline bec1 & 0,592 & $\mathbf{0 , 9 0 7}$ & 0,620 & 0,701 & 0,533 \\
\hline bec2 & 0,651 & $\mathbf{0 , 9 1 4}$ & 0,706 & 0,696 & 0,685 \\
\hline bei1 & 0,720 & 0,728 & $\mathbf{0 , 9 2 7}$ & 0,763 & 0,638 \\
\hline bei3 & 0,835 & 0,616 & $\mathbf{0 , 9 2 0}$ & 0,756 & 0,751 \\
\hline besa1 & 0,587 & 0,652 & 0,547 & $\mathbf{0 , 8 2 2}$ & 0,572 \\
\hline besa2 & 0,772 & 0,640 & 0,730 & $\mathbf{0 , 8 8 9}$ & 0,730 \\
\hline besa6 & 0,784 & 0,655 & 0,794 & $\mathbf{0 , 8 2 5}$ & 0,733 \\
\hline le1 & 0,762 & 0,674 & 0,701 & 0,735 & $\mathbf{0 , 8 5 7}$ \\
\hline le2 & 0,797 & 0,571 & 0,668 & 0,731 & $\mathbf{0 , 8 8 8}$ \\
\hline le3 & 0,755 & 0,547 & 0,649 & 0,682 & $\mathbf{0 , 8 9 3}$ \\
\hline le4 & 0,733 & 0,575 & 0,634 & 0,702 & $\mathbf{0 , 8 9 9}$ \\
\hline
\end{tabular}

Fonte: Resultados da Pesquisa.

Data de Submissão: 18/02/16

Data de Aprovação: 10/05/16 